\title{
Universal Quantum Computation with the Exchange Interaction
}

\author{
D. P. DiVincenzo ${ }^{1}$, D. Bacon ${ }^{2,3}$, J. Kempe ${ }^{2,4,5}$, G. Burkard ${ }^{6}$, and K. B. Whaley ${ }^{2}$ \\ ${ }^{1}$ IBM Research Division, TJ Watson Research Center, Yorktown Heights, NY 10598 USA \\ ${ }^{2}$ Department of Chemistry, University of California, Berkeley, CA 94720 USA \\ ${ }^{3}$ Department of Physics, University of California, Berkeley, CA 94720 USA \\ ${ }^{4}$ Department of Mathematics, University of California, Berkeley, CA 94720 USA \\ ${ }^{5}$ École Nationale Superieure des Télécommunications, Paris, France \\ ${ }^{6}$ Department of Physics and Astronomy, University of Basel, Klingelbergstrasse 82, CH-4056 \\ Basel, Switzerland
}

\begin{abstract}
Experimental implementations of quantum computer architectures are now being investigated in many different physical settings. The full set of requirements that must be met to make quantum computing a reality in the laboratory [i] is daunting, involving capabilities well beyond the present state of the art. In this report we develop a significant simplification of these requirements that can be applied in many recent solid-state approaches, using quantum dots [2], and using donor-atom nuclear spins [3] or electron spins [4]. In these approaches, the basic two-qubit quantum gate is generated by a tunable Heisenberg interaction (the Hamiltonian is $H_{i j}=J(t) \vec{S}_{i} \cdot \vec{S}_{j}$ between spins $i$ and $j$ ), while the one-qubit gates require the control of a local Zeeman field. Compared to the Heisenberg operation, the one-qubit operations are significantly slower and require substantially greater materials and device complexity, which may also contribute to increasing the decoherence rate. Here we introduce an explicit scheme in which the Heisenberg interaction alone suffices to exactly implement any quantum computer circuit, at a price of a factor of three in additional qubits and about a factor of ten in additional two-qubit operations. Even at this cost, the ability to eliminate the complexity of one-qubit operations should accelerate progress towards these solid-state implementations of quantum computation.
\end{abstract}


The Heisenberg interaction has many attractive features [2,5] that have led to its being chosen as the fundamental two-qubit interaction in a large number of recent proposals: Its functional form is very accurate - deviations from the isotropic form of the interaction, arising only from relativistic corrections, can be very small in suitably chosen systems. It is a strong interaction, so that it should permit very fast gate operation, well into the $\mathrm{GHz}$ range for several of the proposals. At the same time, it is very short ranged, arising from the spatial overlap of electronic wavefunctions, so that it should be possible to have an on-off ratio of many orders of magnitude. Unfortunately, the Heisenberg interaction by itself is not a universal gate [6], in the sense that it cannot generate any arbitrary unitary transformation on a collection of spin-1/2 qubits. So, every proposal has supplemented the Heisenberg interaction with some other means of applying independent one-qubit gates (which can be thought of as time-dependent local magnetic fields). But the need to add this capability to the device adds considerably to the complexity of the structures, by putting unprecedented demands on "g-factor" engineering of heterostructure materials [7, [4], requiring that strong, inhomogeneous magnetic fields be applied [2,5], or involving microwave manipulations of the spins that may be slow and may cause heating of the device [4]. These added complexities may well exact a high cost, perhaps degrading the quantum coherence and clock rate of these devices by orders of magnitude.

The reason that the Heisenberg interaction alone does not give a universal quantum gate is that it has too much symmetry: it commutes with the operators $\hat{S}^{2}$ and $\hat{S}_{z}$ (for the total spin angular momentum and its projection on the $z$ axis), and therefore it can only rotate among states with the same $S, S_{z}$ quantum numbers. But by defining coded qubit states, ones for which the spin quantum numbers always remain the same, the Heisenberg interaction alone is universal [8], and single-spin operations and all their attendant difficulties can be avoided.

Recent work has identified the coding required to accomplish this. Starting with early work that identified techniques for suppressing phase-loss mechanisms due to coupling with the environment 11 13, more recent studies have identified encodings that are completely 
immune from general collective decoherence, in which a single environmental degree of freedom couples in the same way to all the spins in a block. These codes are referred to both as decoherence-free subspaces (and their generalization, the decoherence-free subsystems) [14,8, 10], and also as noiseless subspaces and subsystems [15, 16,9]. The noiseless properties of these codes are not relevant to the present work; but they have the desired property that they consist of states with definite angular momentum quantum numbers.

So, in principle, the problem has been solved: the Heisenberg interaction alone is universal and can be used for quantum computation. However, a very practical question still remains: how great is the price that must be paid in return for eliminating single-spin operations? In particular, how many applications of the Heisenberg interaction are needed to complete some desired quantum gate operation? The only guidance provided by the existing theory [8 [10] comes from a theorem of Solovay and Kitaev [17 [19], which states that "efficient" approximations exist: given a desired accuracy parameter $\epsilon$, the number $N$ of exchange operations required goes like $N \approx K \log ^{c}(1 / \epsilon)$, where $c \approx 4$ and $K$ is an unknown positive constant. However, this theorem provides very little useful practical guidance for experiment; it does not show how to obtain the desired approximating sequence of exchange operations, and, since $K$ is unknown, it gives no clue of whether the number of operations needed for a practical accuracy parameter is 10 or 10000. In the following we remedy these inadequacies by showing that the desired quantum logic operations can be obtained exactly using sequences of exchange interactions short enough to be of practical significance for upcoming experiments.

In the scheme we analyze here, we use the smallest subspace with definite angularmomentum quantum numbers that can be used to encode a qubit; this subspace is made up of three spins. It should be noted [10 that in principle the overhead in spatial resources could be made arbitrarily small: asymptotically the rate of encoding into such noiseless subsystems converges to unity. The space of three-spin states with spin quantum numbers $S=1 / 2$, $S_{z}=+1 / 2$ is two dimensional and will serve to represent our coded qubit. A good explicit 
choice for the basis states of this qubit are $\left|0_{L}\right\rangle=|S\rangle|\uparrow\rangle,\left|1_{L}\right\rangle=\sqrt{2 / 3}\left|T_{+}\right\rangle|\downarrow\rangle-\sqrt{1 / 3}\left|T_{0}\right\rangle|\uparrow\rangle$. Here $|S\rangle=\sqrt{1 / 2}(|\uparrow \downarrow\rangle-|\downarrow \uparrow\rangle)$ is the singlet state of spins 1 and 2 (see Fig. 1a) of the three-spin block, and $\left|T_{+}\right\rangle=|\uparrow \uparrow\rangle$ and $\left|T_{0}\right\rangle=\sqrt{1 / 2}(|\uparrow \downarrow\rangle+|\downarrow \uparrow\rangle)$ are triplet states of these two spins. For these states we have constructed an explicit exchange implementation of the basic circuit elements of quantum logic [6]; in particular, we discuss how one obtains any coded one-qubit gate, and a specific two-qubit gate, the controlled NOT (cNOT).

It is easy to understand how one-qubit gates are performed on a single three-spin block. We note that Hamiltonian $H_{12}$ generates a rotation $U_{12}=\exp \left(i / \hbar \int J \vec{S}_{1} \cdot \vec{S}_{2} d t\right)$ which is just a $z$-axis rotation (in Bloch-sphere notation) on the coded qubit, while $H_{23}$ produces a rotation about an axis in the $x-z$ plane, at an angle of $120^{\circ}$ from the $z$-axis. Since simultaneous application of $H_{12}$ and $H_{23}$ can generate a rotation around the $x$-axis, three steps of 1D parallel operation (defined in Fig. 1a) permit any one-qubit rotation, using the classic Euler-angle construction. In serial operation, we find numerically that four steps are always adequate when only nearest-neighbor interactions are possible (eg, the sequence $H_{12^{-}} H_{23^{-}}$ $H_{12}-H_{23}$ shown in Fig. 2a, with suitable interaction strengths), while three steps suffice if interactions can be turned on between any pair of spins (eg, $H_{12}-H_{23}-H_{13}$, see Fig. 2b).

We have performed numerical searches for the implementation of two-qubit gates using a simple minimization algorithm. Much of the difficulty of these searches arises from the fact that while the four basis states $\left|0_{L}, 1_{L}\right\rangle\left|0_{L}, 1_{L}\right\rangle$ have total spin quantum numbers $S=1$, $S_{z}=+1$, the complete space with these quantum numbers for six spins has nine states, and exchanges involving these spins perform rotations in this full nine-dimensional space. So, for a given sequence, eg the one depicted in Fig. 2c, one considers the resulting unitary evolution in this nine-dimensional Hilbert space as a function of the interaction times $t_{1}, t_{2}, \ldots t_{N}$. This unitary evolution can be expressed as a product $U\left(t_{1}, \ldots, t_{N}\right)=U_{N}\left(t_{N}\right) \cdots U_{2}\left(t_{2}\right) U_{1}\left(t_{1}\right)$, where $U_{n}\left(t_{n}\right)=\exp \left(i t_{n} H_{i(n), j(n)} / \hbar\right)$. The objective of the algorithm is to find a set of interaction times such that the total time evolution describes a cNOT gate in the fourdimensional logic subspace $U\left(t_{1}, \ldots, t_{N}\right)=U_{\text {cNOT }} \oplus A_{5}$. The matrix $A_{5}$ can be any unitary 
$5 \times 5$ matrix (consistent with $U$ having a block diagonal form). The efficiency of our search is considerably improved by the use of two invariant functions $m_{1,2}(U)$ identified by Makhlin [20], which are the same for any pair of two-qubit gates that are identical up to one-qubit rotations. It is then adequate to use an algorithm that searches for local minima of the function $f\left(t_{1}, \ldots, t_{N}\right)=\sum_{i}\left(m_{i}\left(U_{\mathrm{cNOT}}\right)-m_{i}\left(U\left(t_{1}, \ldots, t_{N}\right)\right)\right)^{2}$ with respect to $t_{1}, \ldots t_{N}\left(m_{i}\right.$ is understood only to act on the $4 \times 4$ logic subspace of $U$ ). Finding a minimum for which $f=0$ identifies an implementation of cNOT (up to additional one-qubit gates, which are easy to identify [20]) with the given sequence $(i(n), j(n))_{n}, i(n) \neq j(n)$ of exchange gates. If no minimum with $f=0$ is found after many tries with different starting values (ideally mapping out all local minima), we have strong evidence (although not a mathematical proof) that the given sequence of exchange gates cannot generate cNOT.

The optimal serial-operation solution is shown in Fig. 2c. Note that by good fortune this solution happens to involve only nearest neighbors in the 1D arrangement of Fig. 1a. The circuit layout shown obviously has a high degree of symmetry; however, it does not appear possible to give the obtained solution in a closed form. (Of course, any gate sequence involving non-nearest neighbors can be converted to a local gate sequence by swapping the involved qubits, using the SWAP gate, until they are close; here however the minimal solution found does not require such manipulations.) We have also found (apparently) optimal numerical solutions for parallel operation mode. For the 1D layout of Fig. 1a, the simplest solution found involves 8 clock cycles with just $8 * 4$ different interaction-time parameters ( $H_{12}$ can always be zero in this implementation). For the $2 \mathrm{D}$ parallel mode of Fig. 1b, a solution was found using just 7 clock cycles ( $7^{*} 7$ interaction times).

It is worthwhile to give a complete overview of how quantum computation would proceed in the present scheme. It should begin by setting all the computational qubits to the $\left|0_{L}\right\rangle$ state. This state is easily obtained using the exchange interaction: if a strong $H_{12}$ is turned on in each coded block and the temperature made lower than the strength $J$ of the interaction, these two spins will equilibrate to their ground state, which is the singlet state. The third spin in the block should be in the $|\uparrow\rangle$ state, which can be achieved by also placing 
the entire system in a moderately strong magnetic field $B$, such that $k_{B} T<<g \mu_{B} B<J$. Then, computation can begin, with the one- and two-qubit gates implemented according to the schemes mentioned above. For the final qubit measurement, we note that determining whether the spins 1 and 2 of the block are in a singlet or a triplet suffices to perfectly distinguish [7] $\left|0_{L}\right\rangle$ from $\left|1_{L}\right\rangle$ (again, the state of the third spin does not enter). Thus, for example, the AC capacitance scheme for spin measurement proposed by Kane [3] is directly applicable to the coded-qubit measurement.

There are several issues raised by this work that deserve further exploration. The $S=$ $1 / 2, S_{z}=+1 / 2$ three-spin states that we use are a subspace of a decoherence-free subsystem that has been suggested for use in quantum computing by exchange interactions [10,16]. Use of this full subsystem, in which the coded qubit can be in any mixture of the $S_{z}=+1 / 2$ and the corresponding $S_{z}=-1 / 2$ states, would offer immunity from certain kinds of interactions with the environment, and would not require any magnetic field to be present, even for initialization of the qubits. In this modified approach, the implementation of one-qubit gates is unchanged, but the cNOT implementation must satisfy additional constraints the action of the exchanges on both the $S=1$ and the $S=0$ six-spin subspaces must be considered. As a consequence, implementation of cNOT in serial operation is considerably more complex; our numerical studies have failed to identify an implementation (even a good approximate one) for sequences of up to 36 exchanges (cf. 19 in Fig. 2c). On the other hand, we have found implementations using 8 clock cycles for 1D and 2D parallel operation (again for the $1 \mathrm{D}$ case $H_{12}$ can be zero), so use of this larger Hilbert space may well be advantageous in some circumstances.

Finally, we note that further work is needed on the performance of quantum error correction within this scheme. Our logical qubits can be used directly within the error correction codes that have been shown to produce fault tolerant quantum computation [21]. Spin decoherence will primarily result in "leakage" errors, which would take our logical qubits into states of different angular momentum (eg, $S=3 / 2$ ). Our preliminary work indicates that, with small modifications, the conventional error correction circuits will not cause uncon- 
trolled propagation of leakage error. In addition, the general theory [22, 21, 8, 10] shows that there exist sequences of exchange interactions which directly correct for leakage by swapping a fresh $\left|0_{L}\right\rangle$ into the coded qubit if leakage has occurred, and doing nothing otherwise; we have not yet identified numerically such a sequence. If fast measurements are possible, teleportation schemes can also be used in leakage correction.

To summarize, the present results offer a new alternative route to the implementation of quantum computation. The tradeoffs are clear: for the price of a factor of three more devices, and about a factor of ten more clock cycles, the need for stringent control of magnetic fields applied to individual spins is dispensed with. We are hopeful that the new flexibility offered by these results will make easier the hard path to the implementation of quantum computation in the lab. 


\section{REFERENCES}

[1] D. P. DiVincenzo, "The Physical Implementation of Quantum Computation," quantph/0002077, prepared for Fortschritte der Physik special issue, Experimental Proposals for Quantum Computation, to be published.

[2] D. Loss and D.P. DiVincenzo, "Quantum Computation with Quantum Dots," Phys. Rev. A 57, 120-126 (1998).

[3] B.E. Kane, "A Silicon-Based Nuclear-Spin Quantum Computer," Nature 393, 133-137 (1998).

[4] R. Vrijen et al., "Electron-spin-resonance Transistors for Quantum Computing in Silicon-Germanium Heterostructures," Phys. Rev. A 62, 012306 (2000) (10 pages).

[5] G. Burkard, D. Loss and D.P. DiVincenzo, "Coupled Quantum Dots as Quantum Gates," Phys. Rev. B 59, 2070-2078 (1999).

[6] A. Barenco et al., "Elementary Gates for Quantum Computation," Phys. Rev. A 52, 3457-3467 (1995).

[7] D. P. DiVincenzo et al., "Quantum Computation and Spin Electronics," condmat/9911245, prepared for Quantum Mesoscopic Phenomena and Mesoscopic Devices in Microelectronics (eds. I. O. Kulik and R. Ellialtioglu, NATO ASI), to be published.

[8] D. Bacon, J. Kempe, D.A. Lidar and K.B. Whaley, "Universal Fault-Tolerant Computation on Decoherence-Free Subspaces," Phys. Rev. Lett. 85, 1758-1761 (2000).

[9] L. Viola, E. Knill, and S. Lloyd, "Dynamical Generation of Noiseless Quantum Subsystems," quant-ph/0002072.

[10] J. Kempe, D. Bacon, D.A. Lidar and K.B. Whaley, "Theory of Decoherence-Free FaultTolerant Universal Quantum Compuation," submitted to Physical Review A, quantph/0004064. 
[11] W.H. Zurek, "Environment-induced Superselection Rules," Phys. Rev. D 26, 1862-1880 (1982).

[12] G.M. Palma, K.-A. Suominen and A.K. Ekert, "Quantum Computers and Dissipation," Proc. Roy. Soc. London Ser. A 452, 567-584 (1996).

[13] L.-M Duan and G.-C. Guo, "Reducing Decoherence in Quantum-Computer Memory with all Quantum Bits Coupling to the Same Environment," Phys. Rev. A 57, 737-741 (1998).

[14] D.A. Lidar, I.L. Chuang and K. B. Whaley, "Decoherence-Free Subspaces for Quantum Computation," Phys. Rev. Lett. 81, 2594-2597 (1998).

[15] P. Zanardi and M. Rasetti, "Error Avoiding Quantum Codes," Mod. Phys. Lett. B 11, 1085-1093 (1997).

[16] E. Knill, R. Laflamme and L. Viola, "Theory of Quantum Error Correction for General Noise," Phys. Rev. Lett. 84, 2525-2528 (2000).

[17] R. Solovay, unpublished manuscript, 1995.

[18] A. Y. Kitaev, "Quantum Computations: Algorithms and Error Correction," Russ. Math. Surv., 52 (6), 1191-1249 (1997).

[19] M. A. Nielsen and I. L. Chuang, Quantum Computation and Quantum Information (Cambridge University Press, Cambridge, 2000), Appendix 3, "The Solovay-Kitaev Theorem".

[20] Y. Makhlin, "Nonlocal properties of two-qubits Gates and Mixed States and Optimization of Quantum Computations," quant-ph/0002045.

[21] J. Preskill, "Fault-Tolerant Quantum Computation," in Introduction to Quantum Computation and Information (eds. H.-K. Lo, S. Popescu, and T. Spiller, World Scientific, 1998), p. 213-269 quant-ph/9712048. 
[22] D.A. Lidar, D. Bacon, K.B. Whaley, "Concatenating Decoherence-Free Subspaces with Quantum Error Correcting Codes," Phys. Rev. Lett. 82, 4556-4559 (1999).

Acknowledgments: DPD, DB, JK, and KBW acknowledge support from the National Security Agency (NSA) and the Advanced Research and Development Activity (ARDA). DPD also thanks the UCLA DARPA program on spin-resonance transistors for support, and is also grateful for the hospitality of D. Loss at the University of Basel, where much of this work was completed. JK also acknowledges support from the US National Science Foundation. The work of GB is supported in part by the Swiss National Science Foundation. Discussions with P. O. Boykin and B. M. Terhal are gratefully acknowledged. 


\section{FIGURES}
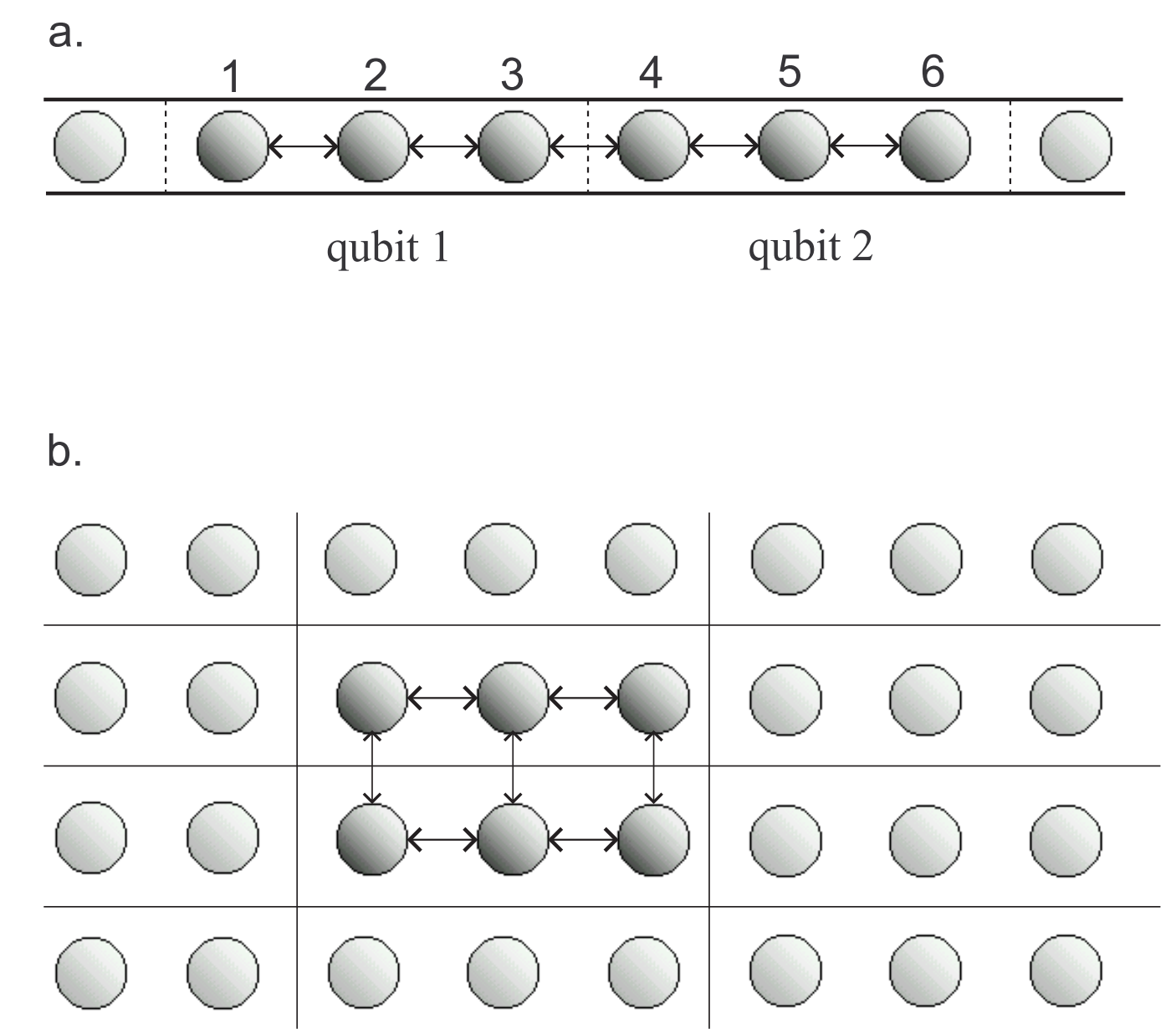

FIG. 1. Possible layouts of spin-1/2 devices. a) One-dimensional layout. We consider two different assumptions about how the exchange interactions can be turned on and off in this layout: 1) At any given time each spin can be exchange-coupled to at most one other spin (we refer to this as "serial operation" in the text), 2) All exchange interactions can be turned on simultaneously between any neighboring pair of spins in the line shown ("1D parallel operation"). b) Possible two-dimensional layout with interactions in a rectangular array. We imagine that any exchange interaction can be turned on between neighboring spins in this array ("2D parallel operation"). Of course other arrangements are possible, but these should be representative of the constraints that will be faced in actual device layouts. 
a.

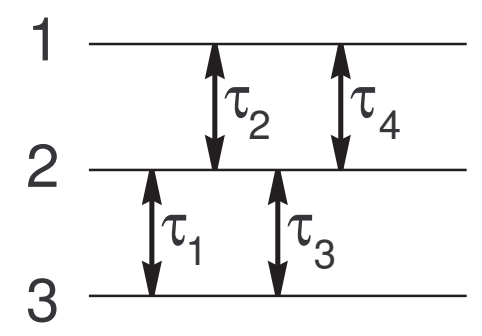

b.

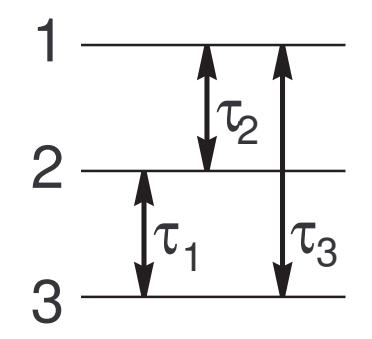

C.

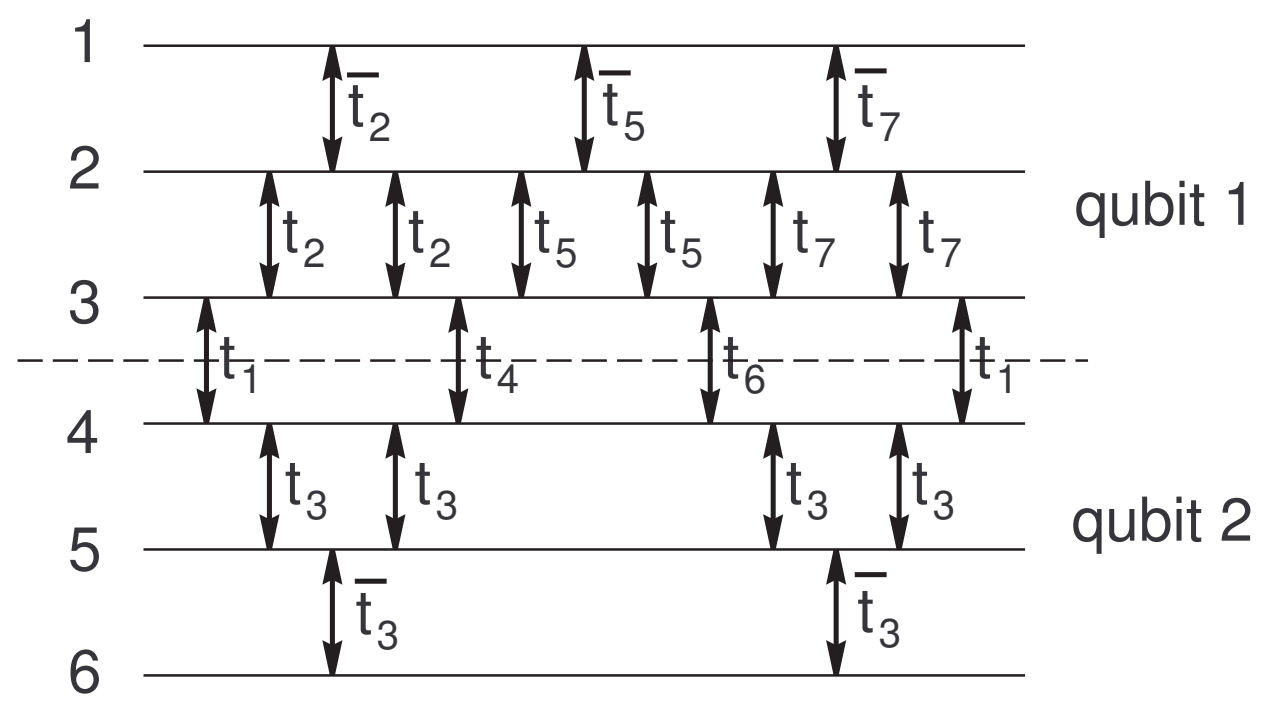

$\mathrm{t}_{1}=0.410899(2) \quad \mathrm{t}_{5}=0.414720(10)$

$\mathrm{t}_{2}=0.207110(20) \quad \mathrm{t}_{6}=0.147654(12)$

$\mathrm{t}_{3}=0.2775258(12) \mathrm{t}_{7}=0.813126(12)$

$\mathrm{t}_{4}=0.640505(8) \quad \tan \left(\pi \mathrm{t}_{\mathrm{i}}\right) \tan \left(\pi \overline{\mathrm{t}}_{\mathrm{i}}\right)=-2$ 
FIG. 2. Circuits for implementing single-qubit and two-qubit rotations using serial operations. a) Single-qubit rotations by nearest-neighbor interactions. Four exchanges (double-headed arrows) with variable time parameters $\tau_{i}$ are always enough to perform any such rotation, one of the two possible layouts is shown. b) Non-nearest neighbor interactions. Only three interactions are needed, one of the possible layouts is shown. c) Circuit of 19 interactions that produce a cNOT between two coded qubits (up to one-qubit gates before and after). The durations of each interaction are given in units such that for $t=1 / 2$ the rotation $U_{i j}=\exp \left(i J t \vec{S}_{i} \cdot \vec{S}_{j} / \hbar\right)$ is a SWAP, interchanging the quantum states of the two spins $i, j$. The $\bar{t}_{i}$ parameters are not independent, they are related to the $t_{i} \mathrm{~s}$ as indicated. The uncertainty of the final digits of these times are indicated in parentheses. With these uncertainties, the absolute inaccuracy of the matrix elements of the two-qubit gate rotations achieved is no greater than $6 \times 10^{-5}$. Further fine tuning of these time parameters would give the cNOT to any desired accuracy. In a practical implementation, the exchange couplings $J(t)$ would be turned on and off smoothly; then the time values given here provide a specification for the integrated value $\int J(t) d t$. The functional form of $J(t)$ is irrelevant, but its integral must be controlled to the precision indicated. The numerical evidence is very strong that the solution shown here is essentially unique, so that no other choices of these times are possible, up to simple permutations and replacements $t \rightarrow 1-t$ (note that for the Heisenberg interaction adding any integer to $t$ results in the same rotation). The results also strongly suggest that this solution is optimal: no one of these 19 interactions can be removed, and no other circuit layout with fewer than 19 has been found to give a solution. We have also sought, but not found, shorter implementations of other interesting two-qubit gates like $\sqrt{\text { SWAP }}$ [2,5]. 